protective Glu149Ala-activated protein C mutant. Blood. 2009;113(23):5970-5978.

8. Guo $\mathrm{H}$, et al. Activated protein $\mathrm{C}$ prevents neuronal apoptosis via protease activated receptors 1 and 3 . Neuron. 2004;41(4):563-572.

9. Yang XV, et al. Activated protein C ligation of ApoER2 (LRP8) causes Dab1-dependent signaling in U937 cells. Proc Natl Acad Sci US A. 2009;106(1):274-279.

10. Cao C, Gao Y, Li Y, Antalis TM, Castellino FJ, Zhang $\mathrm{L}$. The efficacy of activated protein $\mathrm{C}$ in murine endotoxemia is dependent on integrin CD11b. J Clin Invest. 2010;120(6):1971-1980.

11. Kerschen E, et al. Activated protein C targets CD8 dendritic cells to reduce the mortality of endotoxemia in mice. J Clin Invest. 2010;120(9):3167-3178.

12. Elphick GF, et al. Recombinant human activated protein $\mathrm{C}$ inhibits integrin-mediated neutrophil migration. Blood. 2009;113(17):4078-4085.
13. Zheng $X$, et al. Non-hematopoietic EPCR regulates the coagulation and inflammatory responses during endotoxemia. J Thromb Haemost. 2007; 5(7):1394-1400.

14. Shortman K, Naik SH. Steady-state and inflammatory dendritic-cell development. Nat Rev Immunol. 2007;7(1):19-30.

15. Villadangos JA, Shortman K. Found in translation: the human equivalent of mouse CD8+ dendritic cells. J Exp Med. 2010;207(6):1131-1134

16. Hotchkiss RS, Nicholson DW. Apoptosis and caspases regulate death and inflammation in sepsis. Nat Rev Immunol. 2006;6(11):813-822.

17. Xu J, et al. Extracellular histones are major mediators of death in sepsis. Nat Med. 2009;15(11):1318-1321.

18. Kang SJ, Liang HE, Reizis B, Locksley RM. Regulation of hierarchical clustering and activation of innate immune cells by dendritic cells. Immunity.
2008;29(5):819-833.

19. Pereira C, Schaer DJ, Bachli EB, Kurrer MO, Schoedon G. Wnt5A/CaMKII signaling contributes to the inflammatory response of macrophages and is a target for the antiinflammatory action of activated protein C and interleukin-10. Arterioscler Thromb Vasc Biol. 2008;28(3):504-510.

20. Stephenson DA, Toltl LJ, Beaudin S, Liaw PC. Modulation of monocyte function by activated protein C, a natural anticoagulant. J Immunol. 2006; 177(4):2115-2122.

21. Cao C, et al. Endocytic receptor LRP together with tPA and PAI-1 coordinates Mac-1-dependent macrophage migration. EMBO J. 2006;25(9):1860-1870.

22. Nick JA, et al. Recombinant human activated protein $\mathrm{C}$ reduces human endotoxin-induced pulmonary inflammation via inhibition of neutrophil chemotaxis. Blood. 2004;104(13):3878-3885.

\title{
Fitness and freezing: vector biology and human health
}

\section{J. Stephen Dumler}

Division of Medical Microbiology, Department of Pathology, The Johns Hopkins University School of Medicine, Baltimore, Maryland, USA.

\begin{abstract}
Microbes transmitted to mammals by arthropods contend with many factors that could impede survival. To survive, host fitness with infection must outweigh costs. In this issue of the JCI, Neelakanta et al. demonstrate that ticks infected with Anaplasma phagocytophilum show enhanced fitness against freezing injury owing to induced expression of tick "antifreeze glycoprotein." This allows $A$. phagocytophilum to successfully propagate and survive to cause disease in nonnatural hosts, such as humans. How an intracellular microbe with a small genome subverts host cell function for survival provides insight into the control of some cellular function programs and underscores how vector biology can have an impact on human health.
\end{abstract}

Emergence and reemergence of infectious diseases is often attributed to many factors, most of which are influenced by human activities, for example, climate change, environmental change, changes in human demographics and behaviors, and the rise of global trade and travel (1). Perhaps not surprisingly, pathogens evolve or acquire genetic mechanisms that enhance fitness under circumstances of human intervention, such as increasing resistance to potent antimicrobial pharmacologic agents. Emerging infectious disease is most likely to be caused by zoonotic or vector-borne agents (1). This is readily understood for zoonotic and vector-borne RNA viruses (such as tick-borne encephalitis viruses, West Nile virus, and the viruses that cause yellow fever, dengue fever, and Crimean-

Conflict of interest: The author has declared that no conflict of interest exists.

Citation for this article: J Clin Invest. 2010; 120(9):3087-3090. doi:10.1172/JCI44402.
Congo hemorrhagic fever), which undergo frequent genetic change and thus have repeated opportunities to improve fitness (1). However, the substantial evolution of fitness needed to emerge (or reemerge) as a significant health concern in humans is surprising in the context of vector transmission for organisms with less flexible genomes, few opportunities for genetic exchange, and the need to contend with protective mechanisms in at least two distinct hosts. Yet drug-resistant malaria, African and American trypanosomiasis, Lyme disease, and rickettsial infections such as Rocky Mountain spotted fever (RMSF) and human granulocytic anaplasmosis (HGA) have emerged or reemerged to increase in prevalence over the past few decades (2). While considerable investigation is being conducted for some vector-borne emerging diseases and pathogens, bacteria of the order Rickettsiales and their resulting human diseases are understudied, in part because they are obligate intracellular pathogens, which makes biological study difficult, and in part because it is extremely difficult to establish definitive diagnosis, which makes clinical study a challenge (3). Vector biologists who study arthropod-transmitted pathogens understand the role of the vector not only in transmission, but also in disease "ecology." In this issue of the JCI, Neelakanta et al. demonstrate a new paradigm as to how a tick-borne pathogen can manipulate its arthropod host to foster vector survival and indirectly, pathogen transmission, mammalian reservoir maintenance, and, inevitably, human disease (4).

\section{Increasing rickettsial disease prevalence}

While Lyme disease, which is caused by bacteria belonging to the genus Borrelia, is the most common vector-borne human infectious disease in the US (5), rickettsial infections are also increasingly being reported in North America, South America, and Europe, and serological studies show that rickettsial infections are common causes of febrile illness in regions where most fever is attributed to malaria or typhoid fever (5). Recognition of tick-borne rickettsial infections, such as RMSF (caused by Rickettsia rickettsii), African tick-bite fever (caused by Rickettsia africae), human monocytic ehrlichiosis (caused by Ehrlichia chaffeensis), and HGA (caused by Anaplasma phagocytophilum) has markedly expanded in recent years (3). The 4,727 confirmed and unconfirmed reports 


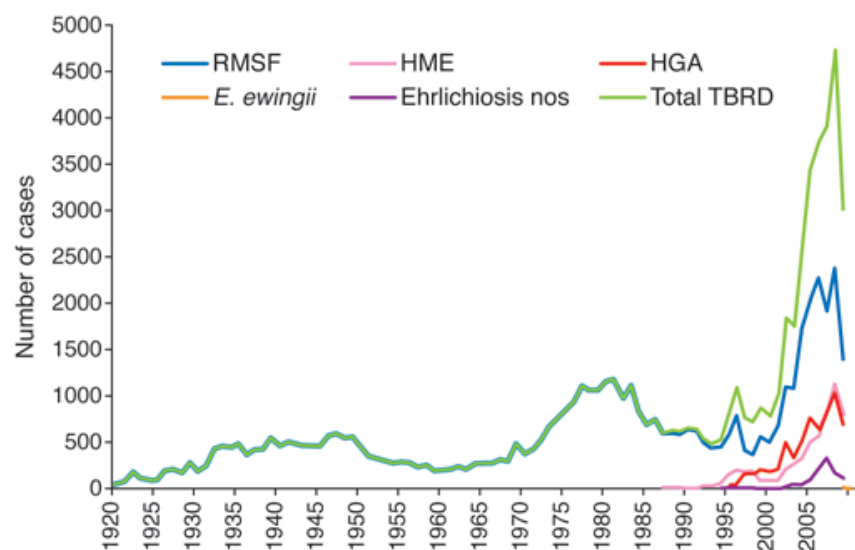

Figure 1

Confirmed and unconfirmed cases of tick-borne rickettsial diseases (TBRD) in the United States, 1920 to 2009. HME, human monocytic ehrlichiosis; nos, not otherwise specified. Graph generated using data obtained from Public Health Service (PHS) and Morbidity and Mortality Weekly Report (MMWR) records (www.CDC.gov/mmwr/).

of tick-borne rickettsial disease to the CDC in 2008 exceed historical highs by a factor of 4 (Figure 1 and ref. 5). Moreover, increasing evidence documents underreporting of RMSF, and likely other tick-borne rickettsial infections, by as much as 5 - to 6 -fold $(6,7)$. These observations parallel those in Brazil, where $R$. rickettsii infection, locally called Brazilian spotted fever, has reemerged, claiming lives in its wake (8). While at least some of the increase in the US is thought to be related to reporting issues (9), the number of additional case reports of humans infected by Ehrlichia spp. and A. phagocytophilum has more than doubled the reported incidence of tick-borne rickettsial infections between the prior peak in 1981 and 2008 (5).

\section{Human disease and obligate intracellular parasites}

Many bacteria in the order Rickettsiales have dual life cycles, one within a mammalian host that amplifies bacterial population size and sometimes provides a safe reservoir from endogenous antimicrobial mechanisms, and one within an arthropod that is the vector for transmission (5). Moreover, their obligatory intracellular lifestyle dictates that they must contend with the substantial challenge of host cell responses to parasitism, even within primary host defense cells such as macrophages and neutrophils and in endothelium, which is continuously surveyed by host defense cells. A. phagocytophilum and Borrelia burgdorferi are both transmitted by Ixodes spp. ticks, and on at least 3 continents, these ticks transmit $A$. phagocytophilum to animals and on occasion to humans.
Human infection with A. phagocytophilum is likely subclinical in the majority of infections, but among those with illness, at least $7 \%$ require intensive care unit admission, and death can occur (10). This is of particular interest because the neutrophil "home" to A. phagocytophilum in human and mammalian hosts is also the primary and most abundant host defense cell. Precisely how A. phagocytophilum survives in this inhospitable environment is predicated on its ability to alter functions of the infected granulocyte, including diminished respiratory burst, enhanced inflammatory recruitment of new host cells, and delayed apoptosis of infected cells $(10,11)$. The ability of the pathogen to alter host cell transcriptional programs seems to be key for each of these phenotypic and functional alterations (12-14). Proven and potential impacts of these alterations are prolonged bacterial survival leading to expanded bacterial populations in the blood of infected mammals, thereby increasing the odds that the next tick bite will result in successful transmission via blood meal acquisition.

\section{Fitness and survival}

The adaptation of $A$. phagocytophilum to the blood niche in a mammal is often subclinical but can result in inflammation and disease. While some aspects of the survival of A. phagocytophilum in mammals are now clear, how it contends with the tick is poorly understood. Around the world, the Ixodes spp. tick vectors of $A$. phagocytophilum, including I. scapularis in North America, I. ricinus in Europe, and I. persulcatus in Asia, inhabit regions where substantial seasonal temperature swings yield freezing conditions for much of the winter. Despite the identification of Ixodes spp. ticks in lower temperate and subtropical latitudes, A. phagocytophilum tick infection and disease prevalence is nearly uniformly restricted to geographic regions that have low average temperatures during winter months (10). Infection of Ixodes spp. ticks by $A$. phagocytophilum was not believed to alter tick fitness under usual study circumstances that assess vector competence in field experiments or laboratories at ambient temperatures maintained well above freezing (15). It is counterintuitive that tick infection, which is mandatory for A. phagocytophilum survival, persists even in the absence of observed direct fitness benefits for the tick. However, in this issue of the JCI, Neelakanta et al. identify a mechanism by which $A$. phagocytophilum enhances tick fitness (4). The hypothesis that Neelakanta and colleagues set to test was that A. phagocytophilum would influence the survival of I. scapularis exposed to freezing temperatures since it survives the winter in tissues of the tick. Exposure of uninfected or A. phagocytophilum-infected ticks to temperatures well below freezing indicated that the infected ticks not only survived better but that survivors were more mobile and therefore more fit. The improved survival of infected ticks correlated with a threshold burden of $A$. phagocytophilum in the tick tissues, supporting a direct link between fitness and bacterial infection. To explain these observations, the authors identified an Ixodes spp. "antifreeze" glycoprotein similar to those found in other arthropods that was upregulated and promoted tick survival during freezing intervals and for which silencing reduced tick survival. Remarkably, the authors were able to demonstrate that infection with $A$. phagocytophilum further enhanced expression of the antifreeze glycoprotein and that this promoted tick fitness by enhancing ability to survive freezing intervals such as those that ticks might experience in the northern latitudes of North America, Europe, or Asia, precisely the areas where the pathogen and infection occur with greatest prevalence. This biological change was mediated by upregulated transcription of the gene encoding the antifreeze glycoprotein. Precisely how A. phagocytophilum accomplishes this transcriptional alteration is unclear, but the ramifications of improving tick fitness for surviving freezing conditions are 


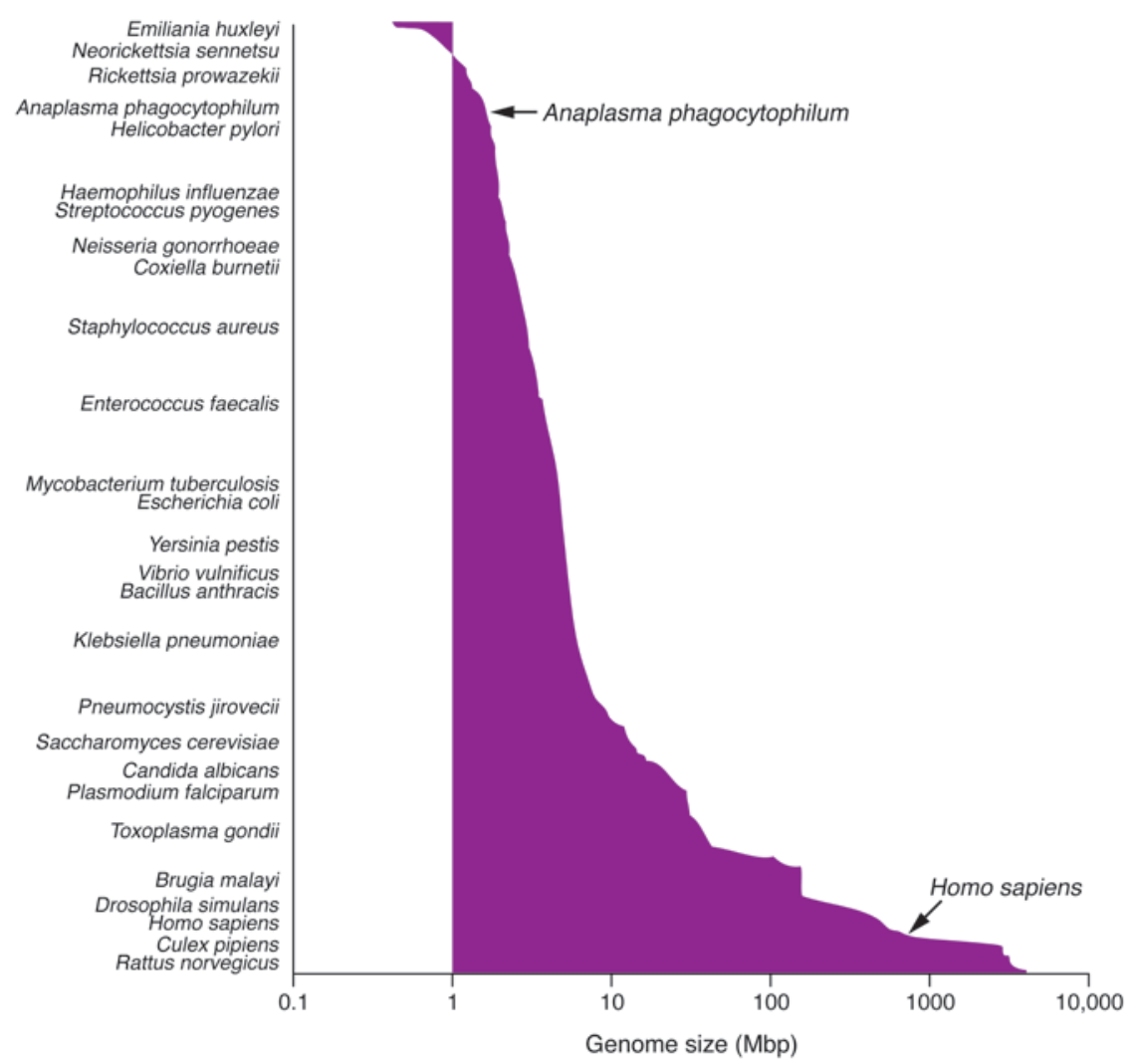

evident in the resulting expansion of bacterial populations and perhaps with the emergence of HGA.

\section{Can pathogen-induced vector fitness have an impact on human health?}

The observations of Neelakanta et al. (4) underscore concepts in vector biology that are generally ignored and often misunderstood by biomedical scientists and the medical community: (a) vector ecology has its basis in biology and (b) the outcomes can weigh substantially on human infection and disease. The understanding of the former concept is best illustrated by examining endosymbionts of arthropods, such as Buchnera aphidicola, a symbiont of plant sap-feeding aphids that require the bacterium for survival because it supplies them with certain amino acids (16); Wigglesworthia spp. mutualists, which promote maternal fecundity, blood meal acquisition, and digestion in Glossina spp. tsetse flies (17); and Wolbachia spp., which infect mosquitoes and regulate iron metabolism and oxidative stress and prevent apoptosis (18).

In the cases of these endosymbionts, manipulation of the host by the microbe often is neutral to the host or mutually beneficial to both microbe and host. However, for those vector-borne microbes whose life cycles require adaptations that promote be detrimental to the vector or to human and animal health. In general, mammalian pathogens transmitted by ticks, fleas, or lice have harmful effects on their vector hosts as well, illustrated in the case of $R$. rickettsii where tick fecundity is decreased, or with Rickettsia prowazekii infection that substantially shortens louse lifespan and therefore reproductive capacity $(19,20)$. In contrast, A. phagocytophilum promotes tick and microbial survival, and thereby, pathogen transmission and dissemination. The ability of A. phagocytophilum to improve tick host fitness, as demonstrated by Neelakanta et al. (4), very likely reflects biological adaptations that further promote microbial survival and transmission in natural mammalian reservoirs. While microbe-induced transcription of the tick antifreeze glycoprotein-encoding gene is unlikely to have an impact on human health directly, the survival adaptations that result from transcriptional reprogramming likely imperil nonnatural, or "nonadapted," accidental hosts such as humans and some animals. Increasing data now demonstrate that A. phagocytophilum has the ability to directly alter host granulocyte antimicrobial and inflammatory transcriptional programs. survival in several hosts, the effects can

\section{Figure 2}

Relative sizes of sequenced eukaryotic, prokaryotic, and Achaea genomes. Graph generated using data available from the NCBI genome database (http://www.ncbi.nlm.nih. gov/sites/genome).

For example, it suppresses transcription of the gene encoding cytochrome b-245, beta polypeptide (CYBB), which is part of the phagocyte oxidase complex that promotes intracellular killing (13), and it induces proinflammatory gene expression that ultimately promotes recruitment of new host cells (21). Both yield microbial survival and expand microbe populations.

A critical question raised by the ability of the microbe to alter fitness with host cell infection is, how does a microbe with one of the smallest genomes control and manipulate cells with 1,000-fold excess genetic reserves (Figure 2)? The most likely scenario involves microbial targeting of host metabolic "bottlenecks" or "weak links," that is, pathways where manipulation alters entire functional programs. This could occur by modifying functional programs governed by epigenetic marks that regulate transcription from large physical regions of host chromatin (12) or by subverting host regulators of broad transcriptional programs, such as NF- $\mathrm{B}$ and CCAAT displacement protein (CDP) $(22,23)$. Disease in humans infected with A. phagocytophilum is truly emerging, but the reasons for this are still under investigation. The work of Neelakanta et al. provides insight into the basic biological processes that belie manipulation of 
the vector by a pathogen, providing a theoretical framework for its impact on human health and disease. These and related findings demonstrate how relevant ecology and vector biology are in protecting human and animal health, especially for emerging zoonotic and vector-borne diseases.

\section{Acknowledgments}

This work was supported in part by NIH/ NIAID R01 AI44102 and R21 AI080911.

Address correspondence to: J. Stephen Dumler, Division of Medical Microbiology, Department of Pathology, 720 Rutland Avenue, Ross Building Room 624, Baltimore, Maryland 21205, USA. Phone: 410.955.8654; Fax: 443.287.3665; E-mail: sdumler@jhmi.edu.

1. Woolhouse ME, Gowtage-Sequeria S. Host range and emerging and reemerging pathogens. Emerg Infect Dis. 2005;11(12):1842-1847.

2. Jones KE, et al. Global trends in emerging infectious diseases. Nature. 2008;451(7181):990-993.

3. Walker DH, Paddock CD, Dumler JS. Emerging and re-emerging tick-transmitted rickettsial and ehrlichial infections. Med Clin North Am. 2008;92(6):1345-1361

4. Neelakanta G, Sultana H, Fish D, Anderson JF, Fikrig E. Anaplasma phagocytophilum induces Ixodes scapularis ticks to express an antifreeze glycoprotein gene that enhances their survival in the cold. J Clin Invest. 2010;120(9):3179-3190.

5. McNabb SJ, et al. Summary of notifiable diseases--
United States, 2006. MMWR Morb Mortal Wkly Rep. 2008;55(53):1-92.

6. Paddock CD, Holman RC, Krebs JW, Childs JE. Assessing the magnitude of fatal Rocky Mountain spotted fever in the United States: comparison of two national data sources. Am J Trop Med Hyg. 2002;67(4):349-354.

7. Paddock CD, et al. Hidden mortality attributable to Rocky Mountain spotted fever: immunohistochemical detection of fatal, serologically unconfirmed disease. J Infect Dis. 1999; 179(6):1469-1476.

8. Angerami RN, et al. Clusters of Brazilian spotted fever in Sao Paulo State, southeastern Brazil. A review of official reports and the scientific literature. Clin Microbiol Infect. 2009;15(suppl 2):202-204.

9. Openshaw JJ, et al. Rocky Mountain spotted fever in the United States, 2000-2007: Interpreting contemporary increases in incidence. Am J Trop Med Hyg. 2010;83(1):174-182.

10. Dumler JS, Madigan JE, Pusterla N, Bakken JS Ehrlichioses in humans: epidemiology, clinical presentation, diagnosis, and treatment. Clin Infect Dis. 2007;45(suppl 1):S45-S51.

11. Carlyon JA, Fikrig E. Mechanisms of evasion of neutrophil killing by Anaplasma phagocytophilum. Curr Opin Hematol. 2006;13(1):28-33.

12. Garcia-Garcia JC, Barat NC, Trembley SJ, Dumler JS. Epigenetic silencing of host cell defense genes enhances intracellular survival of the rickettsial pathogen Anaplasma phagocytophilum. PLoS Pathog. 2009;5(6):e1000488.

13. Garcia-Garcia JC, Rennoll-Bankert KE, Pelly S, Milstone AM, Dumler JS. Silencing of host cell CYBB gene expression by the nuclear effector AnkA of the intracellular pathogen Anaplasma phagocytophilum. Infect Immun. 2009;77(6):2385-2391

14. Borjesson DL, Kobayashi SD, Whitney AR, Voyich JM, Argue CM, Deleo FR. Insights into pathogen immune evasion mechanisms: Anaplasma phagocytophilum fails to induce an apoptosis differen- tiation program in human neutrophils. J Immunol. 2005;174(10):6364-6372.

15. Teglas MB, Foley J. Differences in the transmissibility of two Anaplasma phagocytophilum strains by the North American tick vector species, Ixodes pacificus and Ixodes scapularis (Acari: Ixodidae). Exp Appl Acarol. 2006;38(1):47-58.

16. Brinza L, et al. Systemic analysis of the symbiotic function of Buchnera aphidicola, the primary endosymbiont of the pea aphid Acyrthosiphon pisum. Comptes Rendus Biologies. 2009;332(11):1034-1049.

17. Pais R, Lohs C, Wu Y, Wang J, Aksoy S. The obligate mutualist Wigglesworthia glossinidia influences reproduction, digestion, and immunity processes of its host, the tsetse fly. Appl Environ Microbiol. 2008;74(19):5965-5974.

18. Saridaki A, Bourtzis K. Wolbachia: more than just a bug in insects genitals. Curr Opin Microbiol. 2010;13(1):67-72.

19. Houhamdi L, Fournier PE, Fang R, Lepidi H, Raoult D. An experimental model of human body louse infection with Rickettsia prowazekii. J Infect Dis. 2002;186(11):1639-1646.

20. Niebylski ML, Peacock MG, Schwan TG. Lethal effect of Rickettsia rickettsii on its tick vector (Dermacentor andersoni). Appl Environ Microbiol. 1999;65(2):773-778.

21. Scorpio DG, Akkoyunlu M, Fikrig E, Dumler JS. CXCR2 blockade influences Anaplasma phagocytophilum propagation but not histopathology in the mouse model of human granulocytic anaplasmosis. Clin Diagn Lab Immunol. 2004;11(5):963-968.

22. Choi KS, Scorpio DG, Dumler JS. Anaplasma phagocytophilum ligation to toll-like receptor (TLR) 2, but not to TLR4, activates macrophages for nuclear factor-kappa B nuclear translocation. J Infect Dis. 2004;189(10):1921-1925.

23. Thomas V, Samanta S, Wu C, Berliner N, Fikrig E. Anaplasma phagocytophilum modulates gp91phox gene expression through altered interferon regulatory factor 1 and PU.1 levels and binding of CCAAT displacement protein. Infect Immun. 2005;73(1):208-218.

\title{
Too much PABP, too little translation
}

\author{
Hemant K. Kini, ${ }^{1}$ Melanie R. Vishnu, ${ }^{1}$ and Stephen A. Liebhaber ${ }^{1,2}$
}

${ }^{1}$ Department of Genetics and ${ }^{2}$ Department of Medicine, University of Pennsylvania School of Medicine, Philadelphia, Pennsylvania, USA.

\begin{abstract}
Posttranscriptional regulation is of critical importance during mammalian spermiogenesis. A set of mRNAs that encode proteins critical to normal sperm formation are synthesized early in the process of male germ cell differentiation and are stored in a repressed state. These mRNAs are subsequently translationally activated during the process of spermatid elongation and maturation. Of note, the translationally repressed mRNAs contain long poly(A) tails that are dramatically shortened during the translational activation process. Understanding the mechanisms that underlie this process of mRNA storage and subsequent translational activation has been a long-standing goal. The relationship of the poly(A) tail to translational control is intimately related to the functions of the cognate poly(A)-binding proteins (PABPs). In this issue of the JCI, Yanagiya and colleagues use a set of knockout mice to demonstrate a novel functional role for a particular modulator of PABP function, PABP-interacting protein 2a (PAIP2A), in the normal terminal differentiation of male germ cells.
\end{abstract}

Conflict of interest: The authors have declared that no conflict of interest exists.

Translational control is critical to germline formation

Modulation of mRNA translation is an effective tool to regulate gene expression in the absence of new mRNA synthesis. In part, translation is postulated to be affected by the $5^{\prime}$ methyl-7-guanosine $\left(\mathrm{m}^{7} \mathrm{G}\right)$ cap and $3^{\prime}$ poly(A) tail, two distinguishing features of a typical mature mRNA. Binding of eukaryotic initiation factor $4 \mathrm{E}$ (eIF4E) to the $5^{\prime}$ cap and poly(A)- binding protein (PABP) to the poly(A) tail precedes translational initiation. According to current models, the initiation factor eIF4G can bind simultaneously to both eIF4E and PABP, thus bridging the ends of the transcript. These interactions are proposed to circularize the mRNA, forming a postulated "closed loop" mRNA structure (1). This structure protects the mRNA termini from nuclease attack and enhances translational activity (2). Thus, poly(A) tail length and the poly(A) packaging protein PABP are considered to be critical determinants of both the stability and 